Randomized Controlled Study

\title{
The Efficacy and Safety of the Application of Pulsed Radiofrequency, Combined With Low-Temperature Continuous Radiofrequency, to the Gasserian Ganglion for the Treatment of Primary Trigeminal Neuralgia: Study Protocol for a Prospective, Open- Label, Parallel, Randomized Controlled Trial
}

Hao Ren, MD, Chunmei Zhao, MD, Xiaodi Wang, MD, Ying Shen, MD, Lan Meng, MD, and

Fang Luo, MD

From: Department of Pain Management, Beijing Tiantan Hospital, Capital Medical University, Beijing, China

Address Correspondence: Fang Luo, MD Department of Pain Management, Beijing Tiantan Hospital, Capital Medical University, No. 119 W. Rd, S. 4th Ring Rd, Fengtai District Beijing 100070, Republic of China E-mail: luofangwt@yahoo.com

Disclaimer: This study was supported by the Capital's Funds for Health Improvement and Research (2020-2-2046), the Beijing Municipal Administration of Hospitals Clinical Medicine Development of Special Funding Support (No. XMLX201707) and the Foundation for the Excellent Medical Staff of Beijing (No. 20143-035). The sponsors had no role in the trial design, trial conduct, data handling, data analysis or

writing and publication of the manuscript. HR, CZ and XW contributed equally to this work and should be considered co-first authors.

Conflict of interest: Each author certifies that he or she, or a member of his or her immediate family, has no commercial association (i.e., consultancies, stock ownership, equity interest, patent/licensing arrangements, etc.) that might pose a conflict of interest in connection with the submitted manuscript.

Manuscript received: 12-01-2019 Revised manuscript received: 06-21-2020 Accepted for publication: 07-27-2020

Free full manuscript: www.painphysicianjournal.com
Background: Trigeminal neuralgia is a very painful condition, and radiofrequency therapy is reserved for patients who are resistant or intolerant to pharmacologic therapy. Continuous radiofrequency (CRF) and pulsed radiofrequency (PRF) both have advantages and disadvantages. Recently, studies have found that PRF combined with low-temperature $\left(<65^{\circ} \mathrm{C}\right) \mathrm{CRF}$ increases the efficacy of treatment, without leading to a significant increase in complications caused by nerve lesions. However, these reports have some limitations.

Objectives: We plan to conduct a randomized, controlled study to compare the efficacy of applying high-voltage PRF, with and without low-temperature CRF, to the Gasserian ganglion for the treatment of trigeminal neuralgia.

Study Design: A study protocol for a prospective, open-label, parallel, randomized controlled trial (clinicaltrials.gov; NCT04174443).

Setting: The Department of Pain Management, Beijing Tiantan Hospital, Capital Medical University in Beijing, China.

Methods: One hundred forty-six patients with primary trigeminal neuralgia will be randomly assigned to 1 of 2 groups using an allocation ratio of 1:1. In the high-voltage PRF combined with low-temperature CRF group, $2 \mathrm{~Hz}$ of PRF will be applied under the following conditions: a voltage of $70 \mathrm{~V}$, temperature of $42^{\circ} \mathrm{C}$, pulse width of $20 \mathrm{~ms}$, and treatment time of $600 \mathrm{~s}$. Low-temperature CRF will then be performed at $60^{\circ} \mathrm{C}$, with a treatment time of $270 \mathrm{~s}$. In the high-voltage PRF group, only high-voltage PRF will be performed, using the same treatment parameters. Follow-up process will last for a duration of 1 year.

Results: The primary outcome will be the effectiveness of the treatment after 12 months, which is the percentage of patients with a modified Barrow Neurological Institute Pain Intensity Score (BNI) between I and III. The secondary outcome will include the following: BNI score, Numeric Rating Scale, dose of carbamazepine or oxcarbazepine, patient satisfaction score, quality of life, numbness, side effects, and adverse reactions. These will be recorded over a 1-year follow-up period.

Limitations: The open-label study design may influence the measurement of outcomes and introduce bias, for example, performance or ascertainment bias.

Conclusions: To our knowledge, this will be the first prospective, open-label, parallel, randomized controlled trial to compare the efficacy and safety of the application of high-voltage PRF, combined with and without low-temperature $\left(60^{\circ} \mathrm{C}\right) \mathrm{CRF}$, for the patients who have failed to respond to pharmacologic treatments for primary trigeminal neuralgia. If proven effective, this will be an important, safe, minimally destructive alternative treatment modality for primary trigeminal neuralgia following an ineffective conservative treatment.

Key words: Trigeminal neuralgia, PRF, continuous radiofrequency, high-voltage PRF combined with lowtemperature continuous radiofrequency, study protocol, Barrow Neurological Institute Pain Intensity Score, patient satisfaction score, quality of life, numbness

Pain Physician 2021: 24:89-97 
T rigeminal neuralgia is a very painful condition, characterized by transient and paroxysmal electric, shock-like pain that occurs in areas supplied by the trigeminal nerve (1). The incidence rate of trigeminal neuralgia is approximately 12.6 cases per 100,000 person-years, that increases with age (2). Severe pain has a huge impact on patient's quality of life and often leads to psychological disturbances (3).

Medication is the primary treatment for most patients with trigeminal neuralgia (4). Surgical treatments including microvascular decompression (5), partial sensory rhizotomy (6), radiofrequency therapy $(7,8)$, glycerol rhizolysis $(9,10)$, balloon compression (10), and Gamma knife surgery (11), are reserved for patients who are resistant or intolerant to pharmacologic therapy (4). However, these treatment methods have both advantages and disadvantages; additionally, there is no one ideal surgical treatment yet (4). Continuous radiofrequency (CRF) is a percutaneous radiofrequency procedure that removes the need for endotracheal anesthesia and demands a rather short hospitalization (12-14). CRF also has a very low morbidity and virtually no mortality in comparison to microvascular decompression $(7,15)$. Patients who cannot or prefer not to undergo microvascular decompression or recurrence after craniotomy can choose CRF instead $(4,16)$. Temperature for the application of CRF is usually between $65^{\circ}$ and $80^{\circ}$, and the Gasserian ganglion can thus be ablated through thermal effect $(12,15,17,18)$. Higher temperatures appear to have a greater analgesic effect; however, they result in an increase in complications $(15,19)$. The effectiveness of CRF is inconsistent, nonetheless, most studies suggest that CRF treatment is approximately $70 \%$ to $90 \%$ effective 1 year after the procedure $(12,15,18)$. Although CRF is highly effective, it also results in many severe complications, such as facial numbness, masticatory weakness, and reduced corneal sensation $(20,21)$. The quality of life of patients can be significantly reduced as a result of these complications.

Pulsed radiofrequency (PRF) is one of the most minimally destructive neuromodulation techniques, which can stimulate the Gasserian ganglion using pulsed current $(22,23)$. The temperature of PRF treatment remains below $42^{\circ} \mathrm{C}$, as the heat generated during the $20 \mathrm{~ms}$ of pulsed current has a silent time of $480 \mathrm{~ms}$, during which the heat can dissipate (24). This alludes that it does not cause damage to neural tissue, unlike CRF that can cause nerve damage (22). Patients who receive effective PRF treatment tend to have a more significant improvement in their quality of life than those who receive CRF. However, pure PRF has limited efficacy and a shorter pain relief duration than CRF (16,25-28), even though adjustments have continuously been made to improve this. Our previous research established that the effectiveness of PRF treatment after 1 year could be increased from $41 \%$ to $69 \%$ by increasing the intraoperative output voltage manually $(71.52 \pm 7.97 \mathrm{~V})$, instead of using a standard voltage (29). However, it was still difficult to meet the clinical requirements of efficacy and safety.

Recently, studies have proposed that PRF combined with low-temperature $\left(<65^{\circ} \mathrm{C}\right) \mathrm{CRF}$ could be applied to treat trigeminal neuralgia and may become a promising therapy, increasing the efficacy of treatment without significantly increasing complications due to nerve lesions. Ali Eissa et al (30) retrospectively analyzed the efficacy of PRF, combined with low-temperature $\left(60^{\circ} \mathrm{C}-\right.$ $\left.65^{\circ} \mathrm{C}\right) \mathrm{CRF}$, in 21 patients with trigeminal neuralgia. The effectiveness of treatment measured 1 week and 1 year after the procedure was $71.4 \%$ and $66.7 \%$, respectively. In a randomized controlled study, patients with trigeminal neuralgia received $\mathrm{PRF}, \mathrm{CRF}$ at $75^{\circ} \mathrm{C}$, and PRF combined with low-temperature CRF at $60^{\circ} \mathrm{C}$. The results showed that the effectiveness of treatment in the combined treatment group was $85 \%$, which was higher than that of the single PRF group and CRF group. Side effects were also lower in the combined treatment group than in both PRF and CRF groups (31). In a retrospective study, PRF combined with CRF at $68^{\circ} \mathrm{C}$ was more effective than CRF alone ( $97.5 \%$ vs. $87.5 \%)$ after 1 year, along with fewer side effects and faster recovery times (32). The most recent randomized controlled trial found that PRF, combined with CRF at $60^{\circ} \mathrm{C}$, for the treatment of recurrent trigeminal neuralgia after microvascular decompression, resulted in fewer side effects and complications with a similar effectiveness to CRF at $70^{\circ} \mathrm{C}$ (33). Currently, there is no consensus on the treatment parameters for PRF combined with CRF; including treatment time, temperature, and voltage. Different treatment parameters and outcome indicators may explain significant differences in efficacy reported in different studies.

However, these previous reports were either retrospective or prospective studies with a small sample size and did not disclose some important information such as randomization, blinding method, and sample size calculation. Moreover, to our knowledge, there has been no research on high-voltage PRF combined with lowtemperature CRF. Therefore studies using a larger sample size and a more rigorous clinical design need to be carried 
PRF Combined with CRF for Trigeminal Neuralgia

out to fully evaluate its efficacy. We hypothesize that high-voltage PRF combined with low-temperature CRF is more effective than high-voltage PRF alone. A randomized, controlled study will be conducted to compare the efficacy of high-voltage PRF, combined with and without low-temperature CRF, applied to the Gasserian ganglion for the treatment of trigeminal neuralgia.

\section{Objectives}

To investigate the efficacy and safety of the application of high-voltage PRF combined with lowtemperature $\left(60^{\circ} \mathrm{C}\right)$ CRF to the Gasserian ganglion for the treatment of primary trigeminal neuralgia. We wish to find an effective, safe, and minimally destructive treatment, which can be performed after ineffective conservative treatment.

\section{Methods}

\section{Trial Design}

This will be a prospective, open-label, parallel, randomized controlled trial (Fig. 1).

\section{Setting}

Patients will be recruited from the Department of Pain Management, Beijing Tiantan Hospital, Capital Medical University in Beijing, China. All research members involved in this study will have a prior clinical experience of performing both treatments for at least 1 year. They will be trained according to the same treatment protocol before participating in the study.

This study is scheduled to start in October 2020 and is expected to conclude in a duration of 3 years.

\section{Patients}

\section{Inclusion criteria:}

1. Diagnosed with trigeminal neuralgia according to the International Classification of Headache Disorders, 3rd edition (ICHD-3) criteria (1) (Table 1).

2. Aged 18 to 75 years (inclusive).

3. Suffering from severe trigeminal neuralgia that cannot be alleviated effectively by means of conservative medical therapy, such as carbamazepine, oxcarbazepine.

4. Numeric Rating Scale (NRS-11) score $\geq 7$ before the procedure. 
Table 1. Diagnostic criteria for trigeminal neuralgia in the International Classification of Headache Disorders, 3rd edition (ICHD-3) (1).

\begin{tabular}{|l||}
\hline $\begin{array}{l}\text { A. Recurrent paroxysms of unilateral facial pain in the } \\
\text { distribution(s) of one or more divisions of the trigeminal nerve, } \\
\text { with no radiation beyond, and fulfilling criteria B and C. }\end{array}$ \\
\hline B. Pain has all of the following characteristics: \\
\hline 1. Lasting from a fraction of a second to 2 minutes. \\
\hline 2. Severe intensity. \\
\hline 3. Electric shock-like, shooting, stabbing, or sharp in quality. \\
\hline $\begin{array}{l}\text { C. Precipitated by innocuous stimuli within the affected trigeminal } \\
\text { distribution. }\end{array}$ \\
\hline D. Not better accounted for by another ICHD-3 diagnosis. \\
\hline
\end{tabular}

5. Agreed to sign the informed consent form.

\section{Exclusion criteria:}

1. Secondary trigeminal neuralgia such as trigeminal neuralgia attributed to a space-occupying lesion or multiple sclerosis.

2. Infection at the puncture site.

3. A history of psychiatric disease.

4. Disorder indicated in the results of routine blood tests, hepatic function, renal function, coagulation function, electrocardiogram, or chest x-ray.

5. Serious systemic diseases such as uncontrolled hypertension or diabetes, and cardiac dysfunction (New York Heart Association grade II-III).

6. A history of abuse of narcotics.

7. A history of receiving CRF to the Gasserian ganglion or peripheral branches, glycerol rhizolysis, balloon compression, Gamma knife, or any other neuroablative treatments.

8. A history of receiving microvascular decompression.

\section{Recruitment and Informed Consent}

An experienced attending doctor will enroll patients with primary trigeminal neuralgia at the Department of Pain Management, Beijing Tiantan Hospital, Capital Medical University. All the candidates will be informed in detail of the following information: the purpose of the study, interventions, benefits, possible risks, and corresponding responses. Candidates will be given at least 1 hour to consider whether or not to participate in the study. They will sign the informed consent form voluntarily and will have the right to withdraw from the study at any time without any risk. All of the candidates enrolled in this study will be strictly evaluated, based on the inclusion and exclusion criteria.

\section{Randomization and Blinding}

This will be an open-label trial, so neither the researchers nor the patients will be blinded. Patients will be randomly assigned to a high-voltage PRF combined with low-temperature CRF group, and a highvoltage PRF group at a ratio of 1:1. A simple random sample will be generated by IBM SPSS Statistics 25.0 (IBM Corporation, Armonk, NY). The allocations will be placed in opaque envelopes and sealed. These envelopes will then be placed in a safe, and the key will be safeguarded by the study nurse or the principal investigator. On the day of the procedure, the envelopes will be taken out sequentially according to the order of patients. All of the envelopes will be kept sealed until a puncture has been made in the correct area, and the patients are ready for the initiation of radiofrequency therapy.

\section{Trial Interventions}

All patients will lie in supine position on the computed tomography (CT) scanning table. Blood pressure, heart rate, electrocardiogram, and oxygen saturation will be continuously monitored. The negative plate of the radiofrequency generator (PMG-230, Baylis Medical Inc., Montreal, QC, Canada) will be placed on the skin of the abdomen. The puncture site will then be set approximately $3 \mathrm{~cm}$ from the corner of the mouth on the affected side, using the Hartel technique (34). Local infiltration anesthesia will be administered with $1 \%$ lidocaine. Slice CT scanning ( $2 \mathrm{~mm} / \mathrm{layer}$ ) and 3-dimensional computerized reconstruction will be performed using a CT scanner (LightSpeed 64 slice CT Scanner; General Electric Company, Boston, MA). Under CT guidance, a trocar (100 mm trocar with a 5-mm noninsulated tip, PMF-21-100-5, Baylis Medical Inc., Montreal, QC, Canada) will then be inserted through the ipsilateral foramen ovale to the Gasserian ganglion. The radiofrequency electrode (PMK-21-100, Baylis Medical Inc.) will then take the place of the core of the trocar. Sensory and motor electrical stimulation will be used to adjust the depth and direction of puncturing, to ensure accurate positioning. Fifty hertz of sensory electrical stimulation will induce tingling in the distribution of the trigeminal nerve at 0.1 to $0.3 \mathrm{~V}$ (35). Two hertz of motor electrical stimulation will induce mandibular movement at 0.1 to $0.3 \mathrm{~V}$ (35). After puncture has been made in the correct area, fentanyl $(1 \mu \mathrm{g} / \mathrm{kg}$ ) and midazolam (2 mg) will be given for conscious sedation. The patients will be assigned to either of the following groups, determined by the envelopes opened by the study nurse. 


\section{High-Voltage PRF Combined with Low- Temperature CRF Group}

Two hertz of PRF will be administered at a voltage of $70 \mathrm{~V}$, temperature of $42^{\circ} \mathrm{C}$, pulse width of $20 \mathrm{~ms}$, and treatment time of $600 \mathrm{~s}$. $(29,31)$ Then low-temperature CRF will be performed at $60^{\circ} \mathrm{C}$, with a treatment time of $270 \mathrm{~s}(31,33)$.

\section{High-Voltage PRF Group}

Two hertz of PRF will be administered at a voltage of $70 \mathrm{~V}$, temperature of $42^{\circ} \mathrm{C}$, pulse width of $20 \mathrm{~ms}$, and treatment time of $600 \mathrm{~s}(29,31)$.

Postoperative patients will remain in the hospital for 2 hours to monitor potential complications. The patients will continue to receive oral carbamazepine or oxcarbazepine treatment and the dose of the drug will be reduced gradually according to postoperative pain intensity until its withdrawal. After 1 month, the patients who do not respond well to high-voltage PRF combined with or without low-temperature CRF therapy (those with a modified Barrow Neurological Institute Pain Intensity Score [BNI] of IV and V) will undergo other treatments such as high-temperature CRF, balloon compression, Gamma knife surgery, or microvascular decompression.

\section{Study Outcomes}

Demographic and baseline information will be collected, and will include the following: age, gender, disease duration, laterality, affected nerve branches, NRS-11 scores, doses of carbamazepine or oxcarbazepine given, and scores on the World Health Organization Quality of Life Questionnaire (WHOQOL-BREF).

\section{Primary Outcome}

The modified BNI (Table 2) $(11,36)$ will be used to evaluate the efficacy of treatment. Twelve months after the procedure, patients with BNI scores of I to III will be defined as having received effective treatment. The primary outcome will be the effectiveness of treatment after 12 months.

\section{Secondary Outcome}

- BNI score: BNI scores will be evaluated on day 1 ; after 1 and 2 weeks; and after 1, 2, 3, 6, and 12 months following the procedure.

- NRS-11 score: NRS-11 scores will be evaluated on day 1; after 1 and 2 weeks; and after 1, 2, 3, 6, and 12 months following the procedure.
- Dose of carbamazepine or oxcarbazepine will be recorded on day 1; after 1 and 2 weeks; and after $1,2,3,6$, and 12 months following the procedure.

- Patient satisfaction scores on the 5-point Likert scale (37) (1: poor, 2: fair, 3: good, 4: very good, 5: excellent) will be evaluated after 1,6 , and 12 months following the procedure.

- Quality of life: Scores on the WHOQOL-BREF (38) will be evaluated after 1,6 , and 12 months following the procedure.

- Numbness: BNI facial numbness scores (Table 3) (11) will be evaluated on day 1 ; after 1 and 2 weeks; and after 1, 2, 3, 6, and 12 months following the procedure.

- Anesthesia dolorosa: Whether the patient's facial sensation has decreased and/or is accompanied by pain will be recorded within 12 months of the procedure.

- Masticator weakness: Occlusal dysfunction or a lower degree of masseter muscle fullness on one side when biting hard will be evaluated within 12 months of the procedure.

- Corneal anesthesia: Whether touching the sclera with a sterile cotton swab causes the eyelids to close quickly will be evaluated within 12 months of the procedure.

- Keratitis: Eye dryness and pain, photophobia, lacrimation, excess mucus, and other conditions diagnosed as keratitis will be thoroughly investigated by an ophthalmologist and recorded within 12 months of the procedure.

Table 2. The modified BNI score.

\begin{tabular}{|l|c|}
\hline Score & Pain Relief \\
\hline I & No pain, off medications \\
\hline II & Occasional pain, off medications \\
\hline IIIa & No pain, continued use of medications \\
\hline IIIb & Pain persists, but adequately controlled with medications \\
\hline IV & Pain not adequately controlled with medications \\
\hline V & No relief \\
\hline
\end{tabular}

Table 3. The BNI facial numbness score.

\begin{tabular}{|l|c|}
\hline \hline Score & Pain Relief \\
\hline I & No facial numbness \\
\hline II & Mild facial numbness, not bothersome \\
\hline III & Facial numbness, somewhat bothersome \\
\hline IV & Facial numbness, very bothersome \\
\hline
\end{tabular}


- Adverse reactions: Nausea, vomiting, and facial hematoma during and after the procedure, headache, dizziness, and cerebrospinal fluid leakage occurring within 2 weeks of the procedure will be recorded.

\section{Follow-Up}

The patients will be discharged from the hospital after the procedure. Regular outpatient and telephone follow-ups will be conducted. Outpatient follow-ups will be performed at 1, 6, and 12 months following the procedure, and patients will be suggested to actively report pain recurrence and adverse reactions by telephone or instant messaging. Follow-up will be conducted by a single trained doctor who will not be involved in the patient's enrolment and treatment processes (Table 4).

\section{Data Collection}

Case report forms and standard operating procedure will be based on this research protocol. All the researchers will be systematically trained and will execute a test run before the recruitment process begins. The data required from the case report form will be recorded by the researchers in charge of enrolment and follow-up. The data and safety monitoring committee (DSMC) will monitor the safety and validity data every 6 months to make recommendations on whether or not to continue the study. Back-to-back and double-entry systems using Epidata 4.6 (EpiData Association, Denmark) will ensure the accuracy of data entry.

\section{Safety}

All adverse events will be recorded in detail and given appropriate treatment and follow-up until fully treated or in a stable condition. Serious adverse events will be reported to the ethics committee, competent authorities, and trial sponsors within 24 hours. The DSMC will regularly review all adverse events, and convene meetings when necessary, to assess the risks and benefits of the study. The DSMC has the right to terminate the study at any time.

\section{Patient and Public Involvement}

Neither patients nor the public will be involved in the formulation of research questions, designs, or outcome measurements. Recruitment will be conducted through research posters and physicians' presentations. Patients will be screened and enrolled by trained physicians. The results of this study will be distributed to all patients in the form of newsletters. All patients will be informed regarding relevant intervention in detail.

\section{Sample Size}

The main purpose of this study will be to compare the effectiveness of high-voltage PRF combined with and without low-temperature CRF, evaluated 1 year after the procedure. According to our previous report, the effectiveness of PRF combined with low-temperature CRF group after 1 year was $91 \%$, and that of the high-voltage PRF group was 69\% (29) (The $\alpha$ is 0.05 , and the power is 90\%). Power Analysis and Sample Size (PASS) V.15.0 software (NCSS Corporation, Kaysville, UT, USA) was used to compute the sample size. The results show that 65 patients will be required in each group. However, considering the dropout rate is likely to be $10 \%, 73$ patients will be recruited in each group. Therefore the total sample size of this study will be 146 patients.

\section{Statistical Analysis}

During data entry, names will be replaced by an alphanumeric code, and no personal information will be revealed. The statistician will use both intentionto-treat and per protocol analysis for the dataset using IBM SPSS V25.0 (IBM Corporation). The normally distributed continuous variables will be presented as means \pm standard deviations. The nonnormally distributed continuous variables will be presented as medians (interquartile range). Two-sample t-tests or the MannWhitney/Wilcoxon signed-rank test will be used for data measurement, according to their distributions. The $\chi 2$ test or the Fisher exact test will be used for categorical data. A repeated measures analysis of variance on ranks will be performed for the repeated data, and Bonferroni correction will be used to correct multiple comparisons. Safety assessment will be evaluated using descriptive analysis. A $P$ value of less than an alpha level of 0.05 will be defined as statistically significant.

\section{Ethics and Dissemination}

This protocol was approved by the institutional review board of Beijing Tiantan Hospital (KY-2019-26601) and registered on clinicaltrials.gov (NCT04174443). The trial complies with the guidelines of the Declaration of Helsinki on ethical principles and good clinical practice. All patients will sign the written informed consent prior to randomization. They will also be informed that they have the right to withdraw from the study at any time. The study results will be submitted for publica- 
tion in peer-reviewed journals. The anonymized patient-level dataset will be shared on clinicaltrials.gov.

\section{Discussion}

This study intends to adopt a rigorously designed and implemented randomized controlled method to evaluate the efficacy and safety of the administration of highvoltage PRF, combined with low-temperature CRF, for the treatment of primary trigeminal neuralgia. The aim of this study is to explore a treatment with greater efficacy and fewer side effects for patients with primary trigeminal neuralgia, who have found pharmacologic therapy ineffective. This treatment, if proven effective, may then be used as an alternative to a more traumatic operation.

The trial design of this study is open-label (39), as blinding is considered rather difficult to achieve and strictly maintain. For instance, the difference in radiofrequency treatment time between the 2 groups will make it easy for the health care providers, present to ensure patient safety, to figure out which group the patient has been assigned to. Side effects are also a factor that may cause unintentional unblinding. Additionally, lowtemperature CRF reduces the incidence of facial numbness compared with high-temperature CRF (30-32,40); however, PRF rarely causes facial numbness and other side effects of nerve damage. Therefore some of the patients may be able to determine their group if they experience facial numbness.

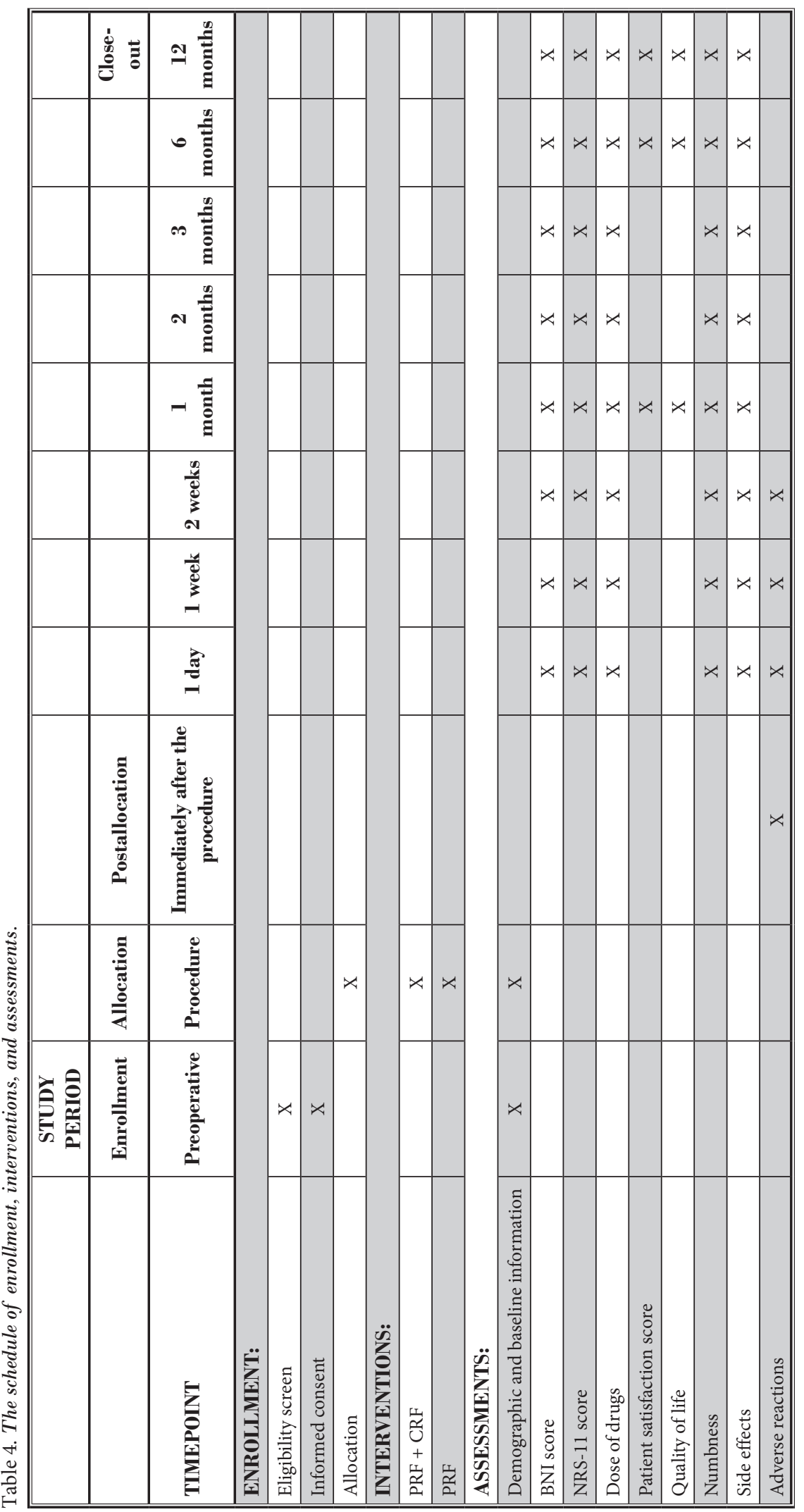


Based on the considerations, we think that if blinding is attempted in this study, it will inevitably lead to inadvertent unblinding. Open-label trials are also extensively used for the evaluation of nonpharmacologic treatments, which are more challenging to blind than pharmacologic treatments $(41,42)$.

BNI score, the primary outcome of this study, will be used to evaluate treatment outcomes and calculate efficacy instead of the NRS-11 or the Visual Analog Scale (VAS). Although the NRS-11 and VAS are the most widely used pain questionnaires, the BNI score has 2 main advantages $(11,36)$ : First, the $\mathrm{BNI}$ score considers both pain and medication. For the patients who do not respond to pharmacologic therapy before the procedure, satisfactory pain relief can still be achieved through medication after radiofrequency procedure. These patients will still have benefitted from radiofrequency treatment. Second, the BNI score assesses the state of the patient, rather than a specific measured value. This makes the definition of the primary outcome clearer and more suitable for an open-label trial (43). Furthermore, we will also be including the NRS-11 to evaluate secondary outcome.

Although it is safer to puncture Gasserian ganglion with a curved blunt needle $(44,45)$, these types of needles are not commercially available in our country. However, the punctures of Gasserian ganglion under the guidance of reconstructed 3-dimensional CT are relatively safe. The correct positioning of the needle tip is key to ensure efficacy and reduce complications. In this study, all the radiofrequency procedures will be performed under CT guidance. CT will clearly show the relative position of the needle as well as bony landmarks, improving the success rate of the puncture. Although CT scans have a higher radiation exposure than a single C-arm scan, CT guidance will allow the puncture to be accomplished more quickly, reducing the risk of additional radiation exposure and potential damage caused by repeated adjustments of needle repositioning (46). We previously reported that the puncture success rate of radiofrequency therapy through the foramen ovale under CT guidance was $100 \%$, and that the punctures caused no severe complications $(8,27)$. Moreover, sensory and motor stimulation will be used to indicate the distance of the needle from the Gasserian ganglion (35). Therefore puncture inaccuracy is highly unlikely to interfere with outcomes.

\section{Conclusions}

There are several limitations of this prospective study. First, the open-label study design may influence the measurement of outcomes and introduce bias, for example performance or ascertainment bias. Second, there is no uniform standard for the treatment duration of PRF and CRF at present. In our study, high-voltage PRF will be administered for $600 \mathrm{~s}$, and low-temperature CRF will be administered for $270 \mathrm{~s}$. This program, along with the treatment voltage and temperature, has been guided by published literatures and our clinical experience. Different treatment parameters may, however, lead to different effects. Finally, we plan to continue the follow-up process for 12 months. Therefore long-term efficacy assessment will require further research.

\section{RefERENCES}

1. Headache Classification Committee of the International Headache Society (IHS) The International Classification of Headache Disorders, 3rd edition. Cephalalgia 2018; 38:1-211.

2. Koopman JS, Dieleman JP, Huygen FJ, et al. Incidence of facial pain in the general population. Pain 2009; 147:122-127.

3. Bonathan CJ, Zakrzewska JM, Love J, et al. Beliefs and distress about orofacial pain: Patient journey through a specialist pain consultation. J Oral Facial Pain Headache 2014; 28:223-232.

4. Bendtsen L, Zakrzewska JM, Abbott J, et al. European Academy of Neurology guideline on trigeminal neuralgia. Eur ] Neurol 2019; 26:831-849.
5. Wang DD, Raygor KP, Cage TA, et al. Prospective comparison of longterm pain relief rates after first-time microvascular decompression and stereotactic radiosurgery for trigeminal neuralgia. J Neurosurg 2018; 128:68-77.

6. Zakrzewska JM, Lopez BC, Kim SE, et al. Patient reports of satisfaction after microvascular decompression and partial sensory rhizotomy for trigeminal neuralgia. Neurosurgery 2005; 56:1304-1311.

7. Kanpolat $Y$, Savas A, Bekar A, et al. Percutaneous controlled radiofrequency trigeminal rhizotomy for the treatment of idiopathic trigeminal neuralgia: 25Year experience with 1,600 patients.
Neurosurgery 2001; 48:524-532.

8. Lan M, Zipu J, Ying S, et al. Efficacy and safety of CT-guided percutaneous pulsed radiofrequency treatment of the Gasserian ganglion in patients with medically intractable idiopathic trigeminal neuralgia. J Pain Res 2018; 11:2877-2885.

9. Udupi BP, Chouhan RS, Dash $\mathrm{HH}$, et al. Comparative evaluation of percutaneous retrogasserian glycerol rhizolysis and radiofrequency thermocoagulation techniques in the management of trigeminal neuralgia. Neurosurgery 2012; 70:407-412.

10. Asplund P, Blomstedt P, Bergenheim AT. Percutaneous balloon compression 
vs percutaneous retrogasserian glycerol rhizotomy for the primary treatment of trigeminal neuralgia. Neurosurgery 2016; 78:421-428; discussion 428.

11. Rogers CL, Shetter AG, Fiedler JA, et al. Gamma knife radiosurgery for trigeminal neuralgia: The initial experience of The Barrow Neurological Institute. Int] Radiat Oncol Biol Phys 2000; 47:1013-1019.

12. Noorani I, Lodge A, Vajramani G, et al. Comparing percutaneous treatments of trigeminal neuralgia: 19 Years of experience in a single centre. Stereotact Funct Neurosurg 2016; 94:75-85.

13. Koopman JSHA, de Vries LM, Dieleman $J P$, et al. A nationwide study of three invasive treatments for trigeminal neuralgia. Pain 2011; 152:507-513.

14. Li Y, Yang L, Ni J, et al. Microvascular decompression and radiofrequency for the treatment of trigeminal neuralgia: A meta-analysis. J Pain Res 2019; 12:1937-1945.

15. Tronnier VM, Rasche D, Hamer J, et al. Treatment of idiopathic trigeminal neuralgia: Comparison of long-term outcome after radiofrequency rhizotomy and microvascular decompression. Neurosurgery 2001; 48:1261-1267.

16. van Kleef M, van Genderen WE, Narouze $S$, et al. 1. Trigeminal neuralgia. Pain Pract 2009; 9:252-259.

17. Sweet WH, Wepsic JG. Controlled thermocoagulation of trigeminal ganglion and rootlets for differential destruction of pain fibers. 1. Trigeminal neuralgia. J Neurosurg 1974; 40:143-156.

18. Broggi $G$, Franzini A, Lasio $G$, et al. Long-term results of percutaneous retrogasserian thermorhizotomy for "essential" trigeminal neuralgia: Considerations in 1000 consecutive patients. Neurosurgery 1990; 26:783-786.

19. Wu H, Zhou J, Chen J, et al. Therapeutic efficacy and safety of radiofrequency ablation for the treatment of trigeminal neuralgia: A systematic review and metaanalysis. J Pain Res 2019; 12:423-441.

20. Bick SKB, Eskandar EN. Surgical treatment of trigeminal neuralgia. Neurosurg Clin N Am 2017; 28:429-438.

21. Cheng JS, Lim DA, Chang EF, et al. A review of percutaneous treatments for trigeminal neuralgia. Neurosurgery 2014; 10(Suppl 1):25-33.

22. Chua NHL, Vissers KC, Sluijter ME. Pulsed radiofrequency treatment in interventional pain management: Mechanisms and potential indications-a review. Acta Neurochir (Wien) 2011; 153:763-771.
23. Van Zundert J, Brabant S, Van de Kelft E, et al. Pulsed radiofrequency treatment of the Gasserian ganglion in patients with idiopathic trigeminal neuralgia. Pain 2003; 104:449-452.

24. Sluijter ME. Non-thermal radiofrequency procedures in the treatment of spinal pain. Pain in Europe; 2nd Annual Congress of the European Federation of IASP Chapters, Barcelona, 1997, pp. 326.

25. Vanneste $T$, Van Lantschoot A, Van Boxem K, et al. Pulsed radiofrequency in chronic pain. Curr Opin Anaesthesiol 2017; 30:577-582.

26. Erdine S, Ozyalcin NS, Cimen A, et al. Comparison of pulsed radiofrequency with conventional radiofrequency in the treatment of idiopathic trigeminal neuralgia. Eur J Pain 2007; 11:309-313.

27. Fang L, Ying S, Tao W, et al. 3D CTguided pulsed radiofrequency treatment for trigeminal neuralgia. Pain Pract 2014; 14:16-21.

28. Sridharan K, Sivaramakrishnan G. Interventions for refractory trigeminal neuralgia: A Bayesian mixed treatment comparison network meta-analysis of randomized controlled clinical trials. Clin Drug Investig 2017; 37:819-831.

29. Fang $L$, Tao $W$, Jingjing $L$, et al. Comparison of high-voltage- with standard-voltage pulsed radiofrequency of Gasserian ganglion in the treatment of idiopathic trigeminal neuralgia. Pain Pract 2015; 15:595-603.

30. Ali Eissa AA, Reyad RM, Saleh EG, et al. The efficacy and safety of combined pulsed and conventional radiofrequency treatment of refractory cases of idiopathic trigeminal neuralgia: A retrospective study. J Anesth 2015; 29:728-733.

31. Elawamy A, Abdalla EEM, Shehata GA. Effects of pulsed versus conventional versus combined radiofrequency for the treatment of trigeminal neuralgia: A prospective study. Pain Physician 2017; 20:E873-E881.

32. Ding $\mathrm{Y}$, Li H, Hong $\mathrm{T}$, et al. Combination of pulsed radiofrequency with continuous radiofrequency thermocoagulation at low temperature improves efficacy and safety in $V_{2} / V_{3}$ primary trigeminal neuralgia. Pain Physician 2018; 21:E545-E553.

33. Abdel-Rahman KA, Elawamy AM, Mostafa MF, et al. Combined pulsed and thermal radiofrequency versus thermal radiofrequency alone in the treatment of recurrent trigeminal neuralgia after microvascular decompression: A double blinded comparative study. Eur J Pain 2020; 24:338-345.
34. Morris L. Trigeminal neuralgia: The anatomy of the "Hartel" technique. The Lancet 1931; 217:122-126.

35. Karol EA, Sanz OP, Rey RD. Sensory and motor trigeminal evoked potentials to localize the position of trigeminal electrodes. Acta Neurochir (Wien) 1991; 108:110-115.

36. McNatt SA, Yu C, Giannotta SL, et al. Gamma knife radiosurgery for trigeminal neuralgia. Neurosurgery 2005; 56:1295-1303.

37. Kianmehr N, Rafati A, Mofidi M, et al. Correlation between pain relief and patient satisfaction. Saudi Med J 2009; 30:1355-1356.

38. World Health Organization. WHOQOLBREF: Introduction, administration, scoring and generic version of the assessment: Field trial version, December 1996. Geneva: World Health Organization, 1996.

39. Sedgwick $P$. What is an open label trial? BM] 2014; 348: 33434 .

40. Arici $T$, Kurcaloglu $M$, Kilic $E$, et al. Radiofrequency thermocoagulation combined with pulsed radiofrequency for gasserian ganglion blockage. Agri 2018; 30:179-182.

41. Boutron I, Tubach F, Giraudeau B, et al. Blinding was judged more difficult to achieve and maintain in nonpharmacologic than pharmacologic trials. J Clin Epidemiol 2004; 57:543-550.

42. Cohen SP, Wallace M, Rauck RL, et al. Unique aspects of clinical trials of invasive therapies for chronic pain. Pain Rep 2019; 4:e687.

43. Kahan BC, Cro S, Doré C), et al. Reducing bias in open-label trials where blinded outcome assessment is not feasible: Strategies from two randomised trials. Trials 2014; 15:456.

44. Taha JM, Tew JM. Comparison of surgical treatments for trigeminal neuralgia: Reevaluation of radiofrequency rhizotomy. Neurosurgery 1996; 38:865-871.

45. Sweet W, Poletti C. Complications of percutaneous rhizotomy and microvascular decompression operations for facial pain. In: Schmideck H (ed). Operative Neurosurgical Techniques: Indication, Methods, and Results. 4th ed. Philadelphia, PA, WB Saunders Co, 2000: pp. 1595-1598.

46. Easwer HVI, Chatterjee N, Thomas A, et al. Usefulness of flat detector CT (FD-CT) with biplane fluoroscopy for complication avoidance during radiofrequency thermal rhizotomy for trigeminal neuralgia. J Neurointerv Surg 2016; 8:830-833. 
